\title{
An Approach for Locating Human Hand Fingers Bone Break from X-beam Pictures
}

\author{
Faiyaz Mohammad Saif \\ Lecturer, CSE \\ European University of \\ Bangladesh
}

\author{
Jabin Rubayat \\ Junior IT Officer \\ Prime Bank Securities Limited \\ Dhaka, Bangladesh
}

\author{
Md.Hosne Al Walid \\ Assistant Professor, CSE \\ Ahsanullah University of \\ Science and Technology
}

\begin{abstract}
Distinguishing human hand fingers bone break is an extremely basic issue in restorative. The framework proposed another approach to recognize these sorts of crack by removing highlights. For doing the general procedure among MRI (Magnetic Resonance Imaging), CT (Computed Tomography) and $\mathrm{X}$-beam pictures, the proposed framework utilized X-beam pictures. At first, the framework takes information from different orthopedic foundations. Subsequent to getting the information the picture preprocessing steps have been done: right off the bat pictures have been changed over into dark, at that point sifted lastly into parallel pictures. From twofold pictures the GLCM (Gray Level Co-event Matrix), minute highlights, entropy, real pivot length, minor hub length, erraticism, introduction, arched region, zone, filled zone, equiv breadth, robustness, degree, border, mean, standard deviation, relationship coefficient, middle, fluctuation, proportion, pixel, and Euclidian separation has been removed. The element esteems are prepared by the Artificial Neural Network (ANN) where the framework used to encourage forward back proliferation systems. At that point, the yield gives two qualities where it is in typical or crack. The figure of prepared Neural Network gives the execution, preparing state, relapse of the trial which is high. The tables decided the gatherings which are changed into size and shapes and furthermore gives that the pictures are in typical or crack with precision $92.24 \%$ which is superior to other. The proposed framework can effectively distinguish the pictures of crack and typical yet can't recognize its composes. Later on, the framework will attempt to test about it.
\end{abstract}

\section{General Terms}

Digital Image Processing, Pattern Recognition

\section{Keywords}

Hand fracture images, x-ray, GLCM, moment feature, neural network, classification.

\section{INTRODUCTION}

The edge of the human hand comprises 27 bones. It has two sections, one is fingers and other is wrists. The wrists are composed into a proximal column and distal row. The four fingers each comprises of three phalanx bones: proximal, center and distal. The thumb just contains proximal and distal phalanx. Correlation with different organs hands bone is the most imperative piece of human body. Without this human is powerless. For the most part amid the blustery and the winter season when after the rain and snowfall the streets wind up pointless for strolling. Particularly for kids and more established individuals, it moves toward becoming dangerous
[1]. Having these different sorts of mishaps like different organs hands bone crack is extremely normal.

A hand crack is a break in one of the bones in anyone's grasp. This incorporates the bones in the wrist and fingers, and those that interface the wrist to the fingers. A handbrake might be caused by contorting or bowing the submit the wrong way. It might likewise be caused by a fall, smash damage or games damage. The accompanying fig. 1 is about the bones of the human hand [2].

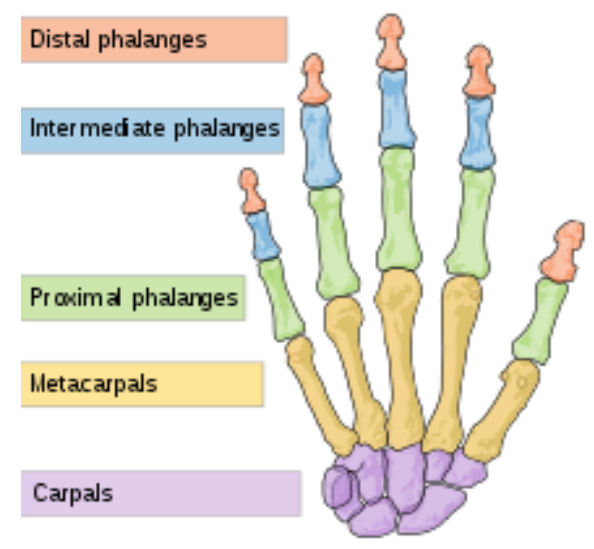

Figure 1: Bones of the Human Hand

The symbols and prefix of a finger fracture are:

- Labor or emotionalism

- Lump or pounding

- Feeling pathetic in passing one's hand

- Unusual bump or unusual shape of one's hand

- Knuckle bone looks submerged in

\section{LITERATURE REVIEW}

There are distinctive kinds of restorative imaging instruments are accessible to recognizing diverse sorts of issues as $\mathrm{X}$ beam, Computed Tomography (CT) and Magnetic Resonance Imaging (MRI) and so on. In any case, for bone break identification X-beam and CT pictures are most as often as possible utilized. For the most part, specialists incline toward the X-beam pictures to distinguish the crack and where it is [3]. Despite the fact that there are a couple of impediments in $\mathrm{X}$-beam however for minimal effort, rapid and ease of use it is extremely powerful.

In past, different bone crack identification strategy is presented as Active Contour demonstrate (ACM and GACM), Wavelet and Haar, Support Vector Machine (SVM) Classifier, $\mathrm{X}$-beam/CT auto characterization of break (GLCM), Novel 
morphological angle based edge location method and can be utilized division or fuzzy knowledge [4] and so on. The bone crack is exceptionally normal in old ages. The typical and irregular pictures have been found and it has been presented in [5]. In [6], the GLCM (Gray Level Co-event Matrix) approach has been utilized to fragment the $\mathrm{x}$-beam pictures of the hand and partitions them separate groups. Subsequent to partitioning the K-implies bunching is utilized for GLCM surface investigation. As a similar way, the GLCM approach has been utilized in[7] where it distinguishes if the break in femur exists or not. Here in the preprocessing steps, the pictures have been converted it into twofold pictures and subsequent to applying edge identification strategy the GLCM based strategies utilized for highlight extraction and perform characterization. Another division approach utilized for $\mathrm{x}-$ pictures of hands in [8] as a base up district blending strategy and furthermore registers mixes between nearby, provincial, worldwide and various leveled separations. In [9], the creators proposed a versatile interface framework considered AdAgen that works together with a prepared specialist. Here utilized neural system to distinguish break in long bones and should be made reference to that their recreation result demonstrate that how NN perform location of the crack in leg radiograph. The best correlation of $\mathrm{x}$-beam picture division strategies has been presented in [10]. The strategies are thresholding, areabased strategies, edge-based division techniques, bunching or group investigation, characterization based division procedures, and level set strategies, Active form models, Active shape models, Wavelet-based systems and knowledgebased methods. Among them, thresholding, edge recognition, grouping based strategies can take care of basic picture division issues yet for complex dynamic form models and dynamic shape models can be utilized. On bone break location there has a work [11] where a crack has been distinguished on estimating the neck shaft edge of the femur. Over that [12], [13], [14] the creators recommend to utilize Gabor, Markov arbitrary field and slope power highlights and feed them into SVM (Support Vector Machine). They likewise demonstrate that these three highlights enhance the exactness of the model. The framework utilized in [15] depicted how the carpal bones can be separated utilizing programmed division techniques. In [16] two procedures have been utilized for deciding skeletal age. One is picture preprocessing utilizing dispersion channel and another is picture division utilizing district level. Another methodology examined in [17] [18] where creators proposed to process the joint width in the $\mathrm{x}$-beam pictures of hands. In [19] proposed a combination characterization procedure for recognizing the break in tibia bone in $\mathrm{x}$-beam pictures.

\section{TYPES OF FINGER FRACTURES}

\subsection{Distal Phalanx Fractures}

Basic kind of distal break is tuft crack. This crack at the fingertip is related with pound damage. In the accompanying figure the bolt sign means the tuft crack as the regular break of distal phalanx crack.

\subsection{Mallet Fracture}

Mallet breaks happen at the inclusion of the terminal finger. It has been caused by a pivotal load to the tip of an expanded finger. The following figure demonstrates the crack where the bone is divided at the dorsal surface of the proximal distal phalanx.

\subsection{Flexor Digit rum Profundus Avulsion Fractures}

A separation crack causes from constrained hyperextensionsof a flexed DIP joint. The accompanying figure demonstrates the break where the bone is divided at the volar surface of the proximal distal phalanx.

\subsection{Center and Proximal Phalanx Fractures}

These cracks are joined with an injury. The breaks are grouped into additional articular where the first is confounded and others are disengaged or found. The accompanying figure demonstrates the proximal phalanx crack of fingers

\section{METHODOLOGY}

In this area the proposed techniques has been set. As talked about before at first pictures has been gathered at that point picture preprocessing steps has been done, at that point GLCM and minute element has been removed at that point order utilizing ANN

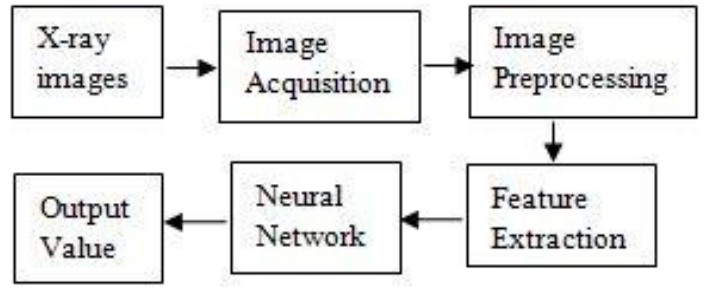

Figure 2: System Architecture of Detecting X-Ray Images

Which strategy is utilized for performing a task that can perceive the pictures with highlights are called includes extraction. It works with a huge arrangement of information or esteem and gives a standard blend with no trouble. In this paper GLCM highlight, Moment includes has been removed.

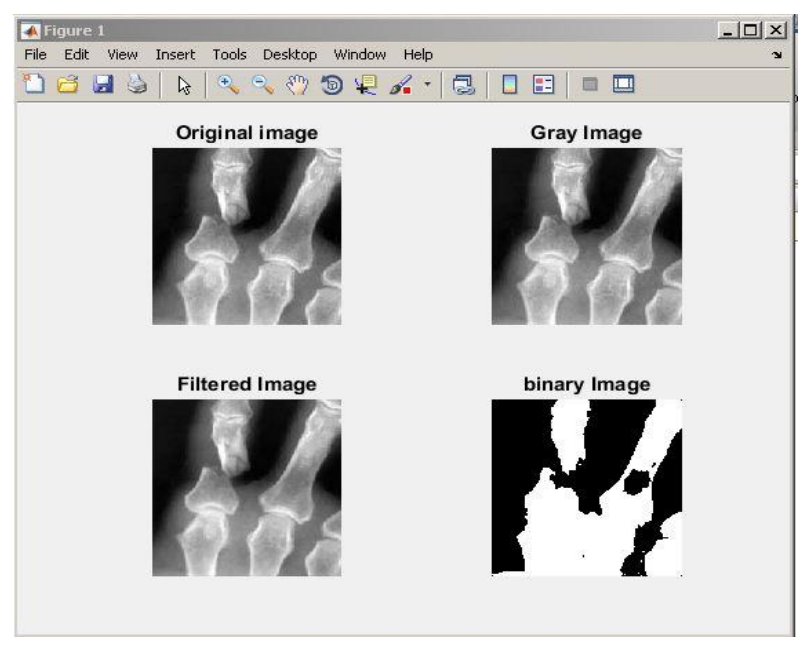

Figure 3: Image Preprocessing

\subsection{GLCM Feature Extraction}

Gray Level Co-event Matrix is a strategy for separating the second request highlights. It requires an extensive number of networks which gives exactness to picture estimation. It likewise speaks to the predefined spatial connection between pixels. The framework utilizes GLCM to get a powerful 
surface from pictures. Graycomatrix work as it used to scaling and to lessen the quantity of force an incentive in a picture to eight. Where the language structure can be [20]:

$$
\text { glcm }=\text { graycomatrix }
$$

Be that as it may, to determine a few measurements shape graycoprops work is utilized for giving data about the surface of a picture. Where the language structure can be:

stats $=$ graycoprops $(\mathrm{glcm}$, properties $)$

details are a structure with fields that are indicated by properties. Graycoprops capacities give data as:

\subsubsection{Contrast}

Returns the force differentiate between a pixel and its neighbor over the between picture.

$$
\text { Contrast }=\sum_{n=0}^{G-1} n^{2}\left\{\sum_{i=1}^{G} \sum_{j=1}^{G} P(i, j)\right\},|i-j|=n
$$

\subsubsection{Correlation}

Returns the estimation of how associated a pixel is to its neighbor over the entomb picture. Range $=\left[\begin{array}{ll}-1 & 1\end{array}\right]$; If Correlation is 1 positive connection if relationship - 1 negative connection if connection is 0 there is no connection between pixel of picture.

$$
\text { Correlation }=\frac{\sum_{i=0}^{N_{g-1}} \sum_{j=0}^{N g-1}(i, j) p(i, j)-\mu_{x} \mu_{y}}{\sigma_{x} \sigma_{y}}
$$

\subsubsection{Energy}

Returns the sum of squared elements in the GLCM. Also known as Angular Second Moment (ASM).

$$
\operatorname{ASM}=\sum_{\mathrm{i}=0}^{\mathrm{N}_{\mathrm{g}-1}} \sum_{\mathrm{j}=0}^{\mathrm{N}_{\mathrm{g}-1}} \mathrm{P}_{\mathrm{ij}}^{2}
$$

\subsubsection{Homogeneity}

Also known as (Inverse Difference Moment) .Returns a value that measures the closeness of the distribution of elements in the GLCM to the GLCM diagonal.

$$
\operatorname{IDM}=\frac{\sum_{\mathrm{i}=0}^{\mathrm{N}_{\mathrm{g}-1}} \sum_{\mathrm{j}=0}^{\mathrm{N}_{\mathrm{g}-1}} \mathrm{P}_{\mathrm{ij}}}{1+(\mathrm{i}-\mathrm{j})^{2}}
$$

\subsection{Moment Feature Extraction}

Ascertain the focal snapshots everything being equal.

SIGMA $=$ moment $(X$, ORDER $)$ restores the ORDER-th focal example snapshot of the qualities in $\mathrm{X}$. For vector input, SIGMA is MEAN((X-MEAN(X))ORDER).

For a lattice input, moment(X,ORDER) restores a line vector containing the focal snapshot of every segment of X. For N-D clusters, minute works along the primary non-singleton measurement. Moment(X,ORDER,DIM) takes the minute along measurement DIM of X [21].
The main focal minute is actually zero. The second focal minute is the fluctuation, utilizing a divisor of $\mathrm{N}$ rather than $\mathrm{N}-1$, where $\mathrm{N}$ is the example measure.

$$
\begin{gathered}
\mathrm{M}_{1}=\eta_{20}+\eta_{02} \\
\mathrm{M}_{2}=\left(\eta_{20}-\eta_{02}\right)^{2}+4 \eta_{11}^{2} \\
\mathrm{M}_{3}=\left(\eta_{30}-3 \eta_{12}\right)^{2}+\left(3 \eta_{21}-\eta_{03}\right)^{2} \\
\mathrm{M}_{4}=\left(\eta_{30}+\eta_{12}\right)^{2}+\left(\eta_{21}+\eta_{03}\right)^{2} \\
\mathrm{M}_{5}=\left(\eta_{30}-3 \eta_{12}\right)\left(\eta_{30}+\eta_{12}\right)\left\lfloor\left(\eta_{30}+\eta_{12}\right)^{2}-\right. \\
3 \eta 21+\eta 032+3 \eta 21-\eta 03 \eta 21+\eta 033 \eta 30+ \\
\eta 032-\eta 21+\eta 032 \\
\mathrm{M}_{6}=\left(\eta_{20}-\eta_{20}\right)\left\lfloor\left(\eta_{30}+\eta_{12}\right)^{2}-\left(\eta_{21}+\eta_{03}\right)^{2}\right\rfloor \\
4 \eta_{11}\left(\eta_{30}+\eta_{12}\right)\left(\eta_{21}+\eta_{03}\right) \\
\mathrm{M}_{7}=\left(3 \eta_{21}-\eta_{03}\right)\left(\eta_{30}+\eta_{12}\right)\left\lfloor\left(\eta_{30}+\eta_{12}\right)^{2}-\right. \\
3 \eta 21+\eta 032-\eta 30+3 \eta 12 \eta 21+\eta 033 \eta 30- \\
\eta 122-\eta 21+\eta 032
\end{gathered}
$$

The seven moments are computed by normalizing central moments with order three.

\subsection{Entropy}

Image entropy is the amount of information which must be coded by a compression algorithm. A perfectly flat image will have zero entropy. It has been computed as:

Entropy $=\sum_{i=0}^{N_{g-1} N_{g-1}} \sum_{j=0}^{N_{g-1}}-P_{i j} * \log P_{i j}$

\subsection{Major Axis Length}

Significant pivot length restores the length (in pixels) of the real hub of the oval that has indistinguishable second-minutes from the locale. It is computed as following:

Major axis $=a+b$

Where $\mathrm{a}, \mathrm{b}$ are the distances from each focus to any point on the ellipse.

\subsection{Minor Axis Length}

Minor axis length returns the length (in pixels) of the minor axis of the ellipse that has the same second-moments as the region. It is measured as:

Minor axis length $=\sqrt{ }\left(\left(a+b^{2}\right)+f^{2}\right)$

Where $\mathrm{f}$ is distance between focus and $\mathrm{a}, \mathrm{b}$ are the distances from each focus to any point on the ellipse.

\subsection{Eccentricity}

Unusualness restores the unconventionality of the circle that has indistinguishable second-minutes from the area. The whimsy is the proportion of the separation between the foci of the oval and its real pivot length. The esteem is somewhere in the range of 0 and 1 . ( 0 and 1 are deteriorating cases; an oval whose unconventionality is 0 is really a circle, while an oval whose unusualness is 1 is a line portion.

\subsection{Orientation}

Orientation returns the angle (in degrees) between the $x$-axis and the major axis of the ellipse that has the same secondmoments as the region. 


\subsection{Convex Area}

Convex area returns a scalar that returns the number of pixels in convex image.

\subsection{Area}

Area returns the actual number of pixels in the region. It can be calculated as:

$$
D=n n z(l(i, j))
$$

Where nnz defines the number of nonzero matrix elements.

\subsection{Filled Area}

It returns the number of pixels in filled image.

\subsection{Equiv Diameter}

Equiv diameter returns a scalar which gives the diameter of a circle with the same area as the region. It is measured as:

$\mathrm{ED}=\sqrt{\frac{4 * \text { Area }}{\mathrm{pi}}}$

\subsection{Solidity}

It returns a scalar that gives the proportion of the pixels in the convex hull that are also in the region. It can be measured as:

$$
\text { Solidity }=\frac{\text { Area }}{\text { Convex area }}
$$

\subsection{Extent}

Extent gives a scalar of the proportion of the pixels in the bounding box that are also in the region. It is measured as:

$$
\text { Extent }=\frac{\text { Area }}{\text { Area of the bounding box }}
$$

\subsection{Perimeter}

The perimeter calculates the distance between each adjoining pair of pixels around the border of the region. If the image contains discontinuous regions, it returns unexpected results. It is measured as:

Distance $={\sqrt{\left(x_{2}-x_{1}\right)^{2}+\left(y_{2}-y_{1}\right)^{2}}}$

\subsection{Mean}

Mean is the average of sum of all the values in the image matrix . It can be calculated as:

Mean $\left.=\operatorname{sum}(A(i, j)) / r^{*} c\right)$

\subsection{Standard Deviation}

Standard deviation is measures of how spread out a distribution is. The variance is computed as the average squared deviation of each number from its mean. Standard deviation is the square root of variance. It has been computed as:

$$
\mathrm{SD}=\sqrt{\text { variance }}
$$

\subsection{Correlation Coefficient}

It returns the value of correlation coefficient between two matrices or vectors of the same size.

\subsection{Median}

It determines the median of the gray scale image.

\subsection{Variance}

This block calculates variance of the input pixels using the following equation.

$$
\text { Variance }=\left(\frac{1}{\mathrm{M} * \mathrm{~N}} \sum_{\mathrm{i}=1}^{\mathrm{M}} \sum_{\mathrm{j}=1}^{\mathrm{N}} \mathrm{X}_{\mathrm{ij}}^{2}\right) \mu^{2}
$$

Ratio for feature has been taken also.

\subsection{Pixel Count}

It gives the percentage of black pixel value of the images that has been calculated as features.

\subsection{Euclidian Distance}

The Euclidean separation is regularly utilized for comparability estimation in picture recovery because of its effectiveness. It checks the separation between two vectors of pictures by figuring the square base of the entirety of the squared supreme contrasts [22]. It tends to be computed as pursues:

$$
E D=\sqrt{ } \sum_{k=1}^{n}\left(x_{1 k}-x_{2 k}\right)^{2}
$$

\section{RESULT ANALYSIS}

In this area, the trial result has been appeared. At first, the proposed framework has been presented as, at that point, the accompanying figures and tables demonstrate the consequences of the analysis

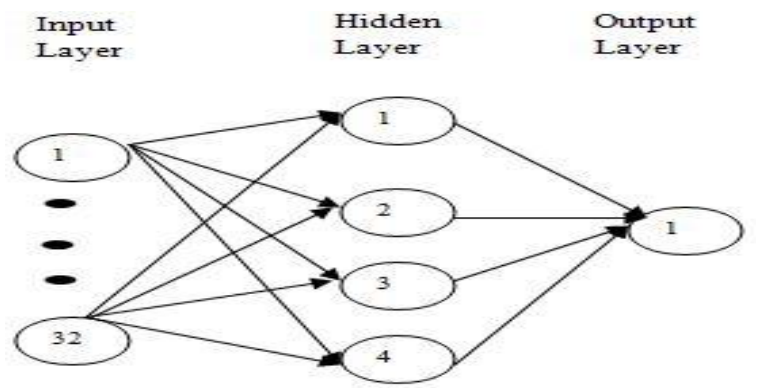

Figure 4: Proposed Network Design

To distinguish hand fingers crack pictures the matlab apparatus has been utilized as it is the high proficient dialect for x-beam pictures. For arrangement Artificial Neural Network (ANN) with back engendering method has been utilized. The framework inclines toward this since it subtracts the preparation yield from the objective (wanted answer) to get the mistake flag. It at that point returns to modify the weights and inclinations in the info and concealed layers to lessen the mistake

For parameter setting the following value is:

Table 1: Parameter Setting

\begin{tabular}{|c|c|}
\hline Parameter & Value \\
\hline Input Layer & 32 \\
\hline Hidden Layer & 4 \\
\hline Output Layer & 1 \\
\hline Learning rate & 0.3 \\
\hline
\end{tabular}


The system used:

Training Class: 02

Targets: 0.4 for Class-1: Normal

Targets: 0.9 for Class-2: Fracture

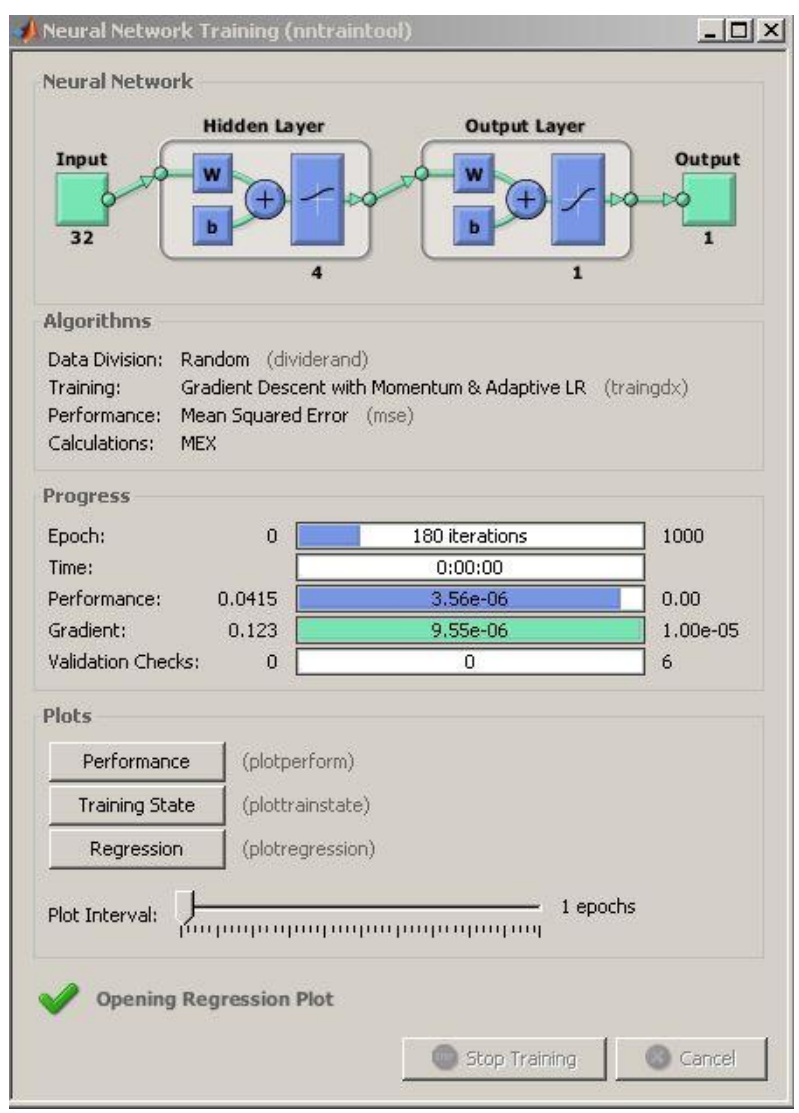

Figure 5: Training Neural Network

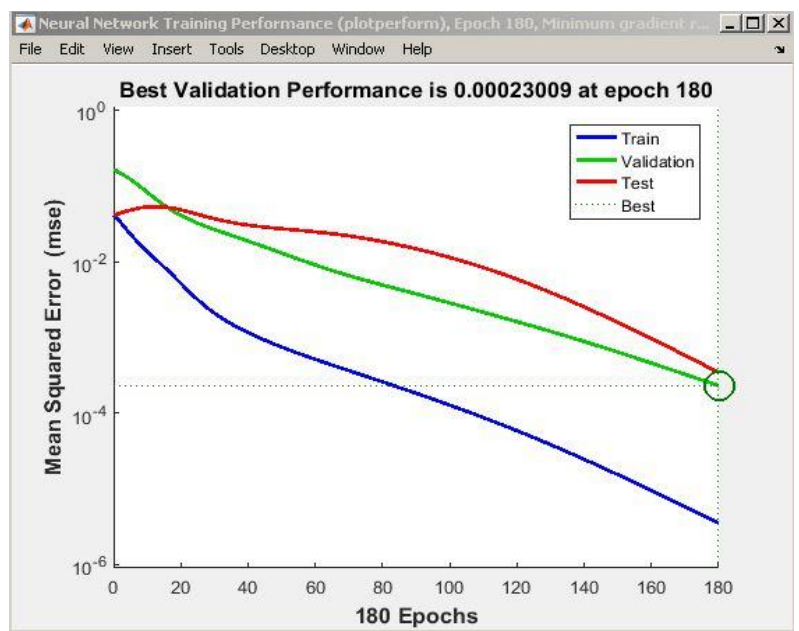

Figure 6: Performance of the Network

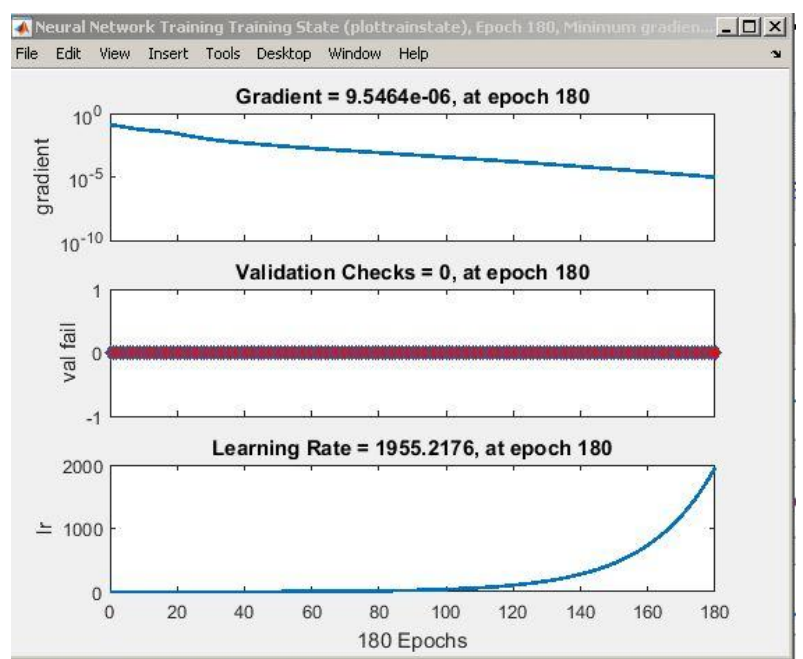

Figure 7: Training State of the network

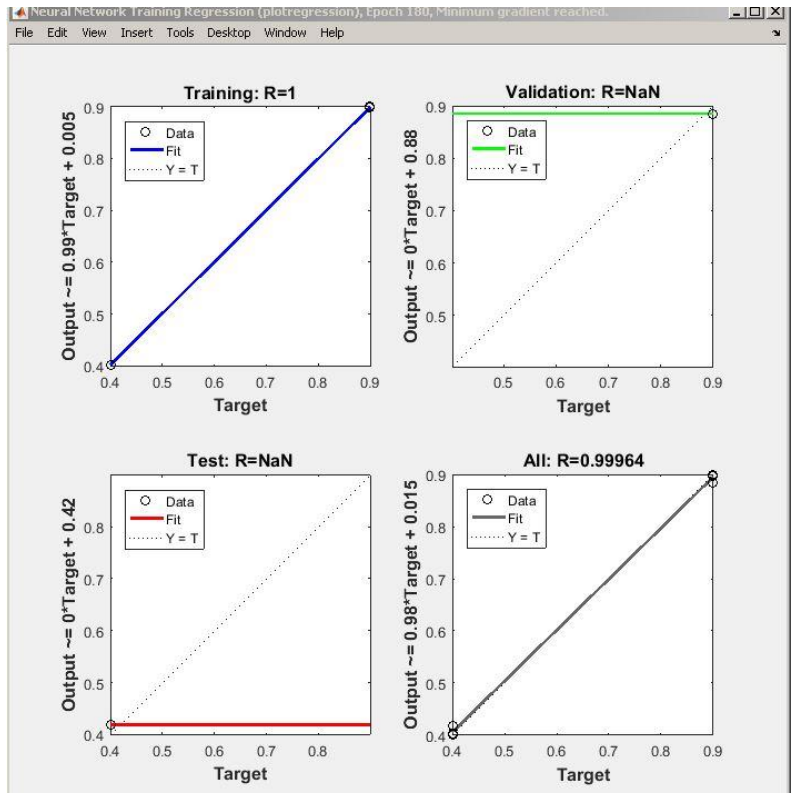

Figure 8: Regression of the Network

The accompanying figures figure $9,10,11,12$ are the screen capture of prepare device, execution, preparing state and relapse of the ANN.A all around prepared ANN ought to have a low MSE (Mean Square Error) toward the finish of the preparation stage which is estimated in the figure of execution plot

Plot regression (targets, yields); plots the linear regression of targets in respect to yields.

Plot prepares state (tr); plots the preparation state from a preparation record tr returned via prepare. 
Table 2: Training with known fracture hand $x$-ray images

\begin{tabular}{|c|c|c|c|c|}
\hline $\begin{array}{c}\text { Image } \\
\text { Type }\end{array}$ & $\begin{array}{c}\text { Number } \\
\text { of image }\end{array}$ & $\begin{array}{c}\text { Correct } \\
\text { detection }\end{array}$ & $\begin{array}{c}\text { Incorrect } \\
\text { detection }\end{array}$ & Accuracy \\
\hline Group-1 & 25 & 23 & 2 & $92 \%$ \\
\hline Group-2 & 15 & 14 & 1 & $93.33 \%$ \\
\hline Group-3 & 20 & 17 & 3 & $85 \%$ \\
\hline Total & 60 & 54 & 6 & $90 \%$ \\
\hline
\end{tabular}

Table 3: Training With Known Normal Hand X-Ray Images

\begin{tabular}{|c|c|c|c|c|}
\hline Image & $\begin{array}{c}\text { Number } \\
\text { of image }\end{array}$ & $\begin{array}{c}\text { Correct } \\
\text { detection }\end{array}$ & $\begin{array}{c}\text { Incorrect } \\
\text { detection }\end{array}$ & Accuracy \\
\hline Group1 & 15 & 15 & 0 & $100 \%$ \\
\hline Group2 & 32 & 30 & 2 & $93.75 \%$ \\
\hline Group3 & 9 & 8 & 1 & $88.89 \%$ \\
\hline Total & 56 & 53 & 3 & $94.64 \%$ \\
\hline
\end{tabular}

Table 4: Compare With Existing System

\begin{tabular}{|c|c|c|}
\hline References & $\begin{array}{l}\text { Existing } \\
\text { System }\end{array}$ & $\begin{array}{l}\text { Accura } \\
\text { cy }\end{array}$ \\
\hline $\begin{array}{c}\text { [1] Al-Ayyoub M and Al-Zghool } \\
\text { D, "Determining the type of long } \\
\text { bone fractures in X-ray images," } \\
\text { WSEAS Transactions on } \\
\text { Information Science and } \\
\text { Applications, 2013;10(8); 261- } \\
70 .\end{array}$ & $\begin{array}{l}\text { Determining } \\
\text { the type of } \\
\text { long bone } \\
\text { fractures in } x- \\
\text { ray images }\end{array}$ & $85 \%$ \\
\hline $\begin{array}{l}\text { [2] Al-Ayyoub M, Hmeidi I, and } \\
\text { Rababah H, "Detecting Hand } \\
\text { Bone Fractures in X-Ray } \\
\text { Images.," JMPT, 2013; 4(3); } \\
\text { 155-68. }\end{array}$ & $\begin{array}{l}\text { Detecting } \\
\text { Hand Bone } \\
\text { Fractures in } \\
\text { X-Ray } \\
\text { Images }\end{array}$ & $91.8 \%$ \\
\hline & $\begin{array}{l}\text { Proposed } \\
\text { System }\end{array}$ & $92.24 \%$ \\
\hline
\end{tabular}

\section{CONCLUSION}

Here the works are about hand crack location utilizing a neural system. Right off the bat acquaint with Computer supported determination which is the best procedure for research region where the frameworks are utilized for improvement [23].

Taking everything into account, Using SVM classifier in order and testing stage the general exactness is over $85 \%$ [18] however utilizing Artificial Neural Network with back spread method in the testing period of this framework the general precision is $92.24 \%$. In spite of the fact that the framework doesn't discover the sorts of hand bone crack however it can accurately distinguish if break exists if or not. To beat this confinement a work will be done in the future and furthermore give the treatment of cracks.

\section{ACKNOWLEDGEMENT}

A big thanks to the respected teachers for their contribution. Necessary help has been taken from internet for overall procedure.

\section{REFERENCES}

[1] Santoso.H. and Nakamura.K., "Situation Awareness Processing Based on Background and Foreground Image for Pedestrian," in SCIS \& ISIS SCIS \& ISIS 2006 , 2006; 949-54.

[2] Ayyoub.A., Hmeidi.M.I. and Rababah.H., "Detecting Hand Bone Fractures in X-Ray Images.," JMPT, 2013; 4(3); 155-68.

[3] Khatik.I., "A Study of Various Bone Fracture Detection Techniques," International Journal of Engineering And Computer Science, 2017; 6(5).

[4] Smith.R., "Segmentation and Fracture Detection in X-ray images for Traumatic Pelvic Injury," 2010.

[5] Miah.M. B. A. and Yousuf.M. A., "Detection of lung cancer from CT image using image processing and neural network," in Electrical Engineering and Information Communication Technology (ICEEICT), 2015 International Conference on, 2015; 1-6.

[6] Chai.H.Y., Wee.L. K., Swee.T.T., Salleh.S.H. and Ariff.A.K., "Gray-level co-occurrence matrix bone fracture detection," American Journal of Applied Sciences, 2011; 8(1); 26.

[7] Chai.H.Y., Wee.L. K., Swee.T.T. and Hussain.S., "GLCM based adaptive crossed reconstructed (ACR) kmean clustering hand bone segmentation," Book GLCM based adaptive crossed reconstructed (ACR) k-mean clustering hand bone segmentation, 2011;192-7.

[8] Lehmann.T.M., Beier.D., Thies.C. and Seidl.T., "Segmentation of medical images combining local, regional, global, and hierarchical distances into a bottomup region merging scheme," in Medical Imaging 2005: Image Processing, 2005; 5747; 546-56.

[9] Syiam.M., El-Aziem.M.A. and El-Menshawy.M., "Adagen: Adaptive interface agent for $\mathrm{x}$-ray fracture detection," International Journal of Computing \& Information Sciences, 2004; 2(3)

[10] Stolojescu.C. and Holban.S., "A comparison of X-ray image segmentation techniques," Advances in Electrical and Computer Engineering, 2013; 13( 3).

[11] Peng.T.T., "Detection of Femur Fractures in X-ray images," Master of Science Thesis, National University of Singapore, 2002.

[12] Lim.S. E., Xing.Y., Chen.Y, Leow.W.K., Howe T. S and Png M. A., "Detection of femur and radius fractures in x-ray images," in Proc. 2nd Int. Conf. on Advances in Medical Signal and Info. Proc, 2004; 65.

[13] Lum.V. L. F., Leow.W. K.., Chen Y., Howe T. S., and Png.M. A., "Combining classifiers for bone fracture detection in X-ray images," in Image Processing, 2005. 
ICIP 2005. IEEE International Conference on, 2005; 1; I1149.

[14] Yap.D., W.H., Chen.Y., Leow.W. K., Howe.T. S., and Png.M. A., "Detecting femur fractures by texture analysis of trabeculae," in Pattern Recognition, 2004. ICPR 2004. Proceedings of the 17th International Conference on, 2004; 3; 730-33.

[15] Hao.S., Han.Y., Zhang.J., and Ji.Z., "Automatic isolation of carpal-bone in hand x-ray medical image," in Informatics and Management Science I, Springer, 2013; 657-62.

[16] Lin.P., Zheng.C., Zhang.F., and Yang.Y., "X-ray carpalbone image boundary feature analysis using region statistical feature based level set method for skeletal age assessment application.," OpticaApplicata, 2005; 35(2).

[17] Bielecki.A., Korkosz.M., Zielinski.B., 'Hand radiographs preprocessing, image representation in the finger regions and joint space width measurements for image interpretation. Pattern Recognition, 2008; 41(12); 378698.
[18] Zielinski.B., "A fully-automated algorithm dedicated to computing metacarpophalangeal and interphalangeal joint cavity widths," SchedaeInformaticae, 2007;16; 47 67.

[19] Mahendran.S., Kand.B. S. S., "An enhanced tibia fracture detection tool using image processing and classification fusion techniques in X-ray images," Global Journal of Computer Science and Technology, 2011; 11(14); 23-28.

[20] Version.M, "9.0. 0 (R2016a)," Math Works Inc., Natick, MA, USA, 2016.

[21] Alam.M.B., "Detection of Brain Cancer from MRI Images using Neural Network.

[22] Al-Amin.M., Miah.M.B.A. and Mia M.R., ".Detection of Cancerous and Non-cancerous Skin busing GLCM Matrix and Neural Network Classifier,' in International Journal of Computer Applications(IJCA),2015,132(8).

[23] Al-Ayyoub.M. and Al-Zghool.D., "Determining the type of long bone fractures in x-ray images," WSEAS Transactions on Information Science and Applications, 2013; 10(8); 261-70. 